\title{
Inclination Measurement of Human Movement Using a 3-D Accelerometer With Autocalibration
}

\author{
Henk J. Luinge and Peter H. Veltink, Member, IEEE
}

\begin{abstract}
In the medical field, accelerometers are often used for measuring inclination of body segments and activity of daily living (ADL) because they are small and require little power. $A$ drawback of using accelerometers is the poor quality of inclination estimate for movements with large accelerations. This paper describes the design and performance of a Kalman filter to estimate inclination from the signals of a triaxial accelerometer. This design is based on assumptions concerning the frequency content of the acceleration of the movement that is measured, the knowledge that the magnitude of the gravity is $1 \mathrm{~g}$ and taking into account a fluctuating sensor offset. It is shown that for measuring trunk and pelvis inclination during the functional three-dimensional activity of stacking crates, the inclination error that is made is approximately $2^{\circ}$ root-mean square. This is nearly twice as accurate as compared to current methods based on low-pass filtering of accelerometer signals.
\end{abstract}

Index Terms-Accelerometer, human, inclination, Kalman filter, kinematics.

\section{INTRODUCTION}

$\mathbf{T}$ HERE are many applications of ambulatory measurement of human movements in the medical field. Inertial sensors, especially accelerometers, are very suitable for these applications because of their small dimensions and weight and the useful movement information they supply. A field in which accelerometers are used is the monitoring of daily life activities (ADL) [1]-[5]. In ADL studies, quantities relating to a subject's energy expenditure, tremor or functional use of a body segment are derived from the accelerometer output. Other applications of accelerometers for human movement recording include the assessment of motor control [6], load estimation using inverse dynamics techniques [7], [8] or artificial sensory feedback for control of electrical neuromuscular stimulation [9]-[11].

A three-dimensional (3-D) accelerometer unit, from now on referred to as an accelerometer, is a transducer that measures acceleration and gravity in three directions. It can be assembled by mounting three single-axis accelerometers in a box with their sensitive axes in different directions or using a sensor based on one mass [12]. Calibrated accelerometers measure the gravity

Manuscript received February 1, 2003; revised April 22, 2003. This work was supported by the Dutch Technology Foundation STW under Contract TEL.4164.

H. J. Luinge was with the Institute of Biomedical Technology, Biomedical Signals and Systems, Department of Electrical Engineering, University of Twente, Enschede $7500 \mathrm{AE}$, the Netherlands. He is now with the Sensory Motor Performance Program, Rehabilitation Institute of Chicago, Chicago, IL 60611 USA (e-mail: luinge@inertia.nl).

P. H. Veltink is with the Institute of Biomedical Technology, Biomedical Signals and Systems, Department of Electrical Engineering, University of Twente,

7500 AE Enschede, the Netherlands (e-mail: p.h.veltink@utwente.nl).

Digital Object Identifier 10.1109/TNSRE.2003.822759 vector and the acceleration in three directions. If the acceleration is small compared to the gravity, the accelerometer can be used as an inclinometer [13]. Accelerometers are frequently used because they are small, robust, relatively cheap, have low power requirements, and can easily be attached to a human body segment. These properties enable the ambulatory monitoring of patients during daily life. No other ambulatory movement sensor to date can match these specifications.

A problem with many accelerometers is that they suffer from a fluctuating offset. This can be due to a temperature change or small changes in the structure (mechanical wear). When accelerometers are to be used in clinical practice a calibration procedure is impractical and can lead to disuse. Lötters et al. proposed an implicit calibration procedure [14]. A high-pass filter was used to determine quasi-static periods in which the subject was standing almost still. Once the accelerometer output was measured at several quasi-static periods in several orientations, the offset and gain could be estimated. This restricts the method to offline analysis. Furthermore, the method only provides proper calibration if a sufficient number of quasi-static periods occur during a trial. A continuous online implicit calibration procedure, not requiring strict quasi-static periods is highly desirable.

Another problem arises when an accelerometer is used as an inclinometer [4], [13]. It requires the acceleration to be sufficiently small in comparison to the gravity. This assumption may be valid under quasi-static conditions like the measurement of sway [15], but is violated during dynamic tasks like lifting [8].

Despite the mentioned drawbacks of accelerometers, they are still the only suitable sensors for long-term ambulatory recording of human body movements. If one 3-D accelerometer could be used to measure an inclination during dynamic tasks without requiring additional sensors, the number of applications will be large. This justifies the current investigation of the maximum amount of information that can be extracted from a 3-D accelerometer.

This study proposes a method to estimate the offset, acceleration, and the gravity vector in a continuous way. It uses a Kalman filter and knowledge about the dynamics of human body movements. The performance of this method was assessed for the daily life task of lifting and stacking objects.

\section{Methods: Filter Design}

\section{A. Accelerometer Signal Components}

In order to estimate offset and inclination from 3-D accelerometer signals, a description of the output of the accelerometer system is required. In the current study, the 


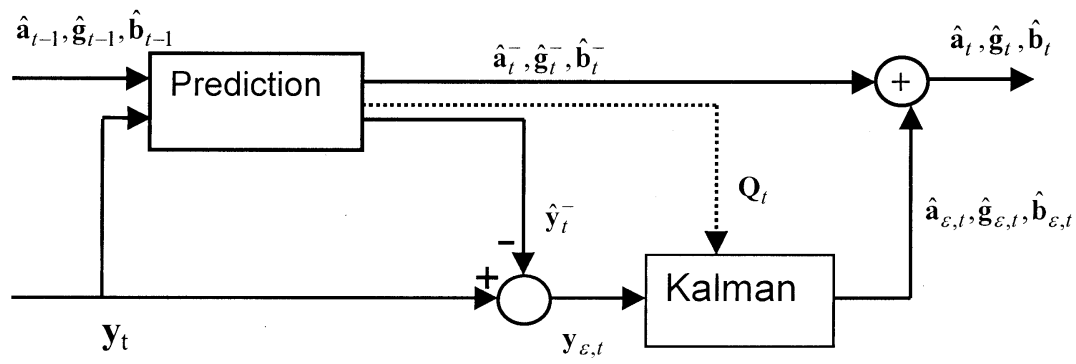

Fig. 1. Structure of the Kalman filter. Previous estimates of the acceleration, gravity and offset $\left(\hat{\mathbf{a}}_{t}^{-}, \hat{\mathbf{g}}_{t}^{-}, \hat{\mathbf{b}}_{t}^{-}\right)$are used to predict the sensor output vector. The difference $\left(\mathbf{y}_{\varepsilon, t}\right)$ between predicted sensor output $\left(\hat{\mathbf{y}}_{t}^{-}\right)$and actual sensor output $\left(\mathbf{y}_{t}\right)$ is a function of the prediction error of the acceleration, gravity, and offset. A Kalman filter attributes this difference to the prediction errors $\left(\hat{\mathbf{a}}_{\varepsilon, t}, \hat{\mathbf{g}}_{\varepsilon, t}, \hat{\mathbf{b}}_{\varepsilon, t}\right)$ using the variances of the predicted components $\left(\mathbf{Q}_{t}\right)$. A hat on top of a symbol is used to indicate an estimate, the minus sign an a priori estimate on the basis of the signal model and a plus sign an a posteriori estimate after correction by the Kalman filter.

3-D accelerometer output is assumed to be composed of an acceleration, a gravity, and an offset vector with inevitable superimposed measurement noise

$$
\mathbf{y}_{t}=\mathbf{a}_{t}-\mathbf{g}_{t}+\mathbf{b}_{t}+\mathbf{v}_{A, t} \text {. }
$$

All accelerometer output signal components, the acceleration $\mathbf{a}_{t}$, the gravity $\mathbf{g}_{t}$, the offset $\mathbf{b}_{t}$, and white noise $\mathbf{v}_{A, t}$ are expressed in the sensor coordinate frame. The offset $\mathbf{b}_{t}$ is a component that may change slowly with time and is considered unknown at the start of an experiment. Possible disturbances due to nonlinear contributions and fluctuations of gain are assumed to have a minor effect on the sensor output and are, therefore, not incorporated in the model.

Knowing the direction of the gravity vector in the sensor coordinate frame, the inclination can be calculated. To obtain the inclination, the different components of (1) have to be separated. For this purpose, a Kalman filter was designed that uses the characteristics of the different accelerometer output components.

\section{B. Description of Estimation Procedure}

On the basis of a model of the accelerometer signal, a discrete-time complementary Kalman filter [16], [17] was designed to estimate the acceleration, offset, and gravity. The estimation procedure is as follows (Fig. 1): An a priori prediction of the accelerometer signal components offset, gravity, and acceleration, and their covariances is made on the basis of the previously estimated values and the sensor signal. This prediction is then compared to the actual sensor output. The Kalman filter uses an error model in state-space format that relates the prediction error to errors in the a priori offset and gravity estimates. On the basis of this error model and covariances of the estimated sensor components, the difference between predicted and actual sensor output is attributed to $a$ priori prediction errors.

First, a model is presented that describes the accelerometer signals, based on the characteristics of the components. This model is used to estimate the different signal components. Subsequently, the covariances of these components are derived, on the basis of which the Kalman filter can attribute the prediction error to these components.

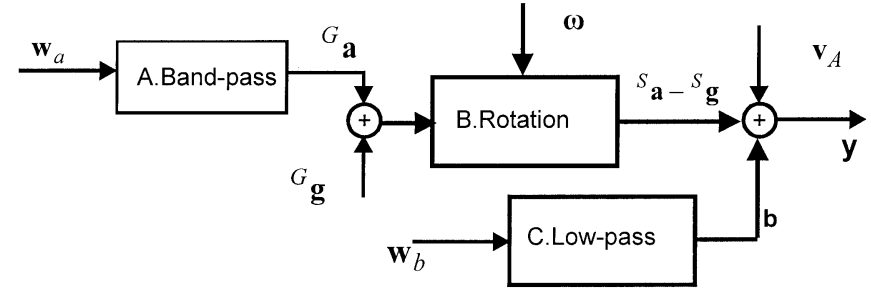

Fig. 2. Model of the different components that constitute the accelerometer signal. $\mathbf{w}_{a}$ and $\mathbf{w}_{b}$ are white noise signals. ${ }^{G} \mathbf{a}$ denotes the acceleration in an earth fixed reference frame. The sensor output is modeled as the acceleration minus gravity ${ }^{S} \mathbf{a}-{ }^{S} \mathbf{g}$ expressed in the accelerometer coordinate frame that is rotating with an angular velocity $\boldsymbol{\omega}$, plus a white measurement noise component $\mathbf{v}_{A}$ and slowly varying offset $\mathbf{b}$. The lower cutoff frequency of the bandpass filter of block $\mathrm{A}$ is much higher than the lowpass cutoff frequency of block $\mathrm{C}$.

\section{Accelerometer Signal Generation Model}

The model describing the accelerometer signals is based on the following assumptions.

1) The bandwidth of the acceleration is limited because of the inertia of the body segment on which the sensor is attached.

2) The acceleration of a body segment expressed in a nonrotating coordinate frame has zero mean. Even a small acceleration in a particular direction for longer than a few seconds would result in an unrealistic displacement and speed of the human segment. This means that the acceleration spectrum is zero for low frequencies.

3) The bandwidth of the offset vector is low with respect to the bandwidth of the acceleration.

A block diagram of the model is given in Fig. 2. The acceleration $G \mathbf{a}$ in a global coordinate frame was modeled as a realization of a bandpass filtered white zero-mean noise (block A in Fig. 2, assumption 1 and 2). The accelerometer measures the acceleration minus gravity vector in a coordinate frame that is rotating with an angular velocity $\boldsymbol{\omega}$ with respect to an earth-fixed coordinate frame (block B, rotation). A white noise signal $\mathbf{v}_{A}$ and the slowly varying offset $\mathbf{b}$ are added to form the accelerometer signal. (block C, assumption 3).

The acceleration was modeled as an AR process (block A in Fig. 2, assumption 1 and 2)

$$
{ }^{G} \mathbf{a}_{t}=\mathbf{H}(q)\left\{{ }^{G} \mathbf{a}_{t}\right\}+{ }^{G} \mathbf{w}_{a, t}
$$


where $\mathbf{w}_{a}$ is a white noise vector and $\mathbf{H}$ a polynomial function, using the backward shift operator q. The left superscript $\mathrm{G}$ denotes a vector expressed in the global coordinate frame. Because of assumptions 1 and 2, a bandpass characteristic is to be expected. Equation (2) was used to predict the acceleration. Since the acceleration is not measured in the global frame but in the sensor frame, the acceleration expressed in the current sensor frame was used for the prediction. The unknown noise term ${ }^{G} \mathbf{w}_{a, t}$ was set to zero

$$
{ }^{S, t} \hat{\mathbf{a}}_{t}^{-}=\mathbf{H}(q)\left\{{ }^{S, t} \hat{\mathbf{a}}_{t-1}^{+}\right\}
$$

A hat on top of a symbol denotes an estimation and the minus superscript denotes the a priori estimation of a signal component before it is corrected with the Kalman filter. A plus superscript denotes an estimation that is made after correction with the Kalman filter and the $S, t$ on the left of a vector means that the vector is expressed in the sensor coordinate frame at time $t$.

The gravity remains the same vector in a nonrotating coordinate frame. Therefore, the direction of the new gravity estimate equals the old and the magnitude is renormalized to $1 \mathrm{~g}$. $\mathrm{g}_{\mathrm{C}}$ equals the gravitational constant

$$
{ }^{S, t} \hat{\mathrm{g}}_{t}^{-}=g_{\mathrm{C}} \cdot \frac{S, t \hat{\mathrm{g}}_{t-1}^{+}}{\left|S,{ } \hat{\mathrm{g}}_{t-1}^{+}\right|}
$$

Equations (3) and (4) express the predicted acceleration and gravity at previous instances in the sensor coordinate frame of the current timestep. The vectors at previous sensor frames have to be rotated in order to be expressed in the current sensor frame. Relation (5) describes the transformation of the acceleration and gravity from the previous sensor coordinate frame $(S, t-1)$ to the current sensor coordinate frame $(S, t)$ by rotating the vectors over a small angle given by the angular velocity $\boldsymbol{\omega}$ and sample time $T$

$$
{ }^{S, t} \mathbf{r}={ }^{S, t-1} \mathbf{r}-T \boldsymbol{\omega} \times{ }^{S, t-1} \mathbf{r} .
$$

The angular velocity is estimated from the raw accelerometer signal, as described in Section III.

The angular velocity is estimated by considering the rate of change of the accelerometer output. If the accelerometer would measure a constant and nonrotating vector in an earth-fixed coordinate frame, the angular velocity would cause a change in the measured vector according to (5). Since, for many types of human movement, the gravity vector is a large component of the sensor signal vector, a rough estimation of the angular velocity vector can be made. The component of the angular velocity perpendicular to the measured vector is used as an estimate of angular velocity and can be written explicitly according to

$$
\hat{\boldsymbol{\omega}}_{t}=\frac{1}{T} \cdot \mathbf{n}_{t-1} \times \mathbf{n}_{t}
$$

with $\mathbf{n}$ the unit vector in the direction of the measured accelerometer signal vector $\mathbf{y}_{t}$.

The angular velocity prediction in the direction of $\mathbf{y}_{t}$ can not be assessed by an accelerometer and was, therefore, set to zero. The accuracy of the angular velocity estimate perpendicular to $\mathbf{y}_{t}$ depends on the validity of the assumption that the change in acceleration is small with respect to the magnitude of the sensor signal vector.

To obtain an expression describing the accuracy of (6) consider a sensor signal that changes during a time interval $T$ because of a change in acceleration $\Delta \mathbf{a}$ and a sensor rotation with an angular velocity $\boldsymbol{\omega}$

$$
{ }^{S, t} \mathbf{y}_{t}={ }^{S, t-1} \mathbf{y}_{t-1}+{ }^{S, t} \Delta \mathbf{a}_{t}-T \boldsymbol{\omega}_{t} \times{ }^{S, t-1} \mathbf{y}_{t-1}
$$

The error in the angular velocity estimate, $\boldsymbol{\omega}_{\varepsilon}=\hat{\omega}-\boldsymbol{\omega}$, can be found by substituting the expression for the change in accelerometer output vector (7) into the angular velocity estimation (6)

$$
\begin{aligned}
\boldsymbol{\omega}_{\varepsilon, t} & =-\left(\boldsymbol{\omega}_{t} \cdot \mathbf{n}_{t-1}\right) \mathbf{n}_{t-1}+\frac{1}{g_{c}} \mathbf{n}_{t-1} \times \Delta \mathbf{a}_{t} \\
& =\boldsymbol{\omega}_{\|, t}+\boldsymbol{\omega}_{\varepsilon \perp, t} .
\end{aligned}
$$

The two terms on the right-hand side of (8) are the angular velocity parallel and perpendicular to the accelerometer signal vector, respectively. Since this vector will mostly point approximately in the gravity direction, the angular velocity around the vertical will generally be unknown.

The angular velocity prediction only requires accelerometer signals and can, therefore, be obtained before processing the accelerometer signals with the Kalman filter. To diminish the error caused by the second term on the right-hand side of (8), the estimated angular velocity is low-pass filtered.

The offset is modeled as a Markov process according to (block C Fig. 2, assumption 3)

$$
\mathbf{b}_{t}=\mathbf{b}_{t-1}+\mathbf{w}_{b, t} \text {. }
$$

A prediction of the offset is made by setting the white noise vector $\mathbf{w}_{b}$ to zero.

\section{Error Model}

The Kalman filter uses a state space structure that describes the effect of prediction errors in the acceleration, gravity, and offset on the difference in predicted and measured accelerometer vector

$$
\begin{aligned}
& \mathbf{x}_{\varepsilon, t}=\mathbf{A} \cdot \mathbf{x}_{\varepsilon, t-1}+\mathbf{w}_{t} \\
& \mathbf{y}_{\varepsilon, t}=\mathbf{C} \cdot \mathbf{x}_{\varepsilon, t}-\mathbf{v}_{t}
\end{aligned}
$$

$\mathbf{y}_{\varepsilon, t}$ is the difference between the predicted and measured accelerometer output. This difference is a result from errors in predicted offset, gravity, and acceleration. The error state $\mathbf{x}_{\varepsilon, t}$ is the state that is estimated by the Kalman filter. Since the goal of this paper is to estimate the inclination and offset, these two vectors were included in the state vector $\mathbf{x}_{\varepsilon}$

$$
\mathbf{x}_{\varepsilon, t}=\left[\begin{array}{ll}
\mathbf{g}_{\varepsilon, t} & \mathbf{b}_{\varepsilon, t}
\end{array}\right]^{T}
$$

$\mathbf{w}_{t}$ and $\mathbf{v}_{t}$ are white noise signals with covariance matrices $\mathbf{Q}$ and $\mathbf{R}$, respectively. $\mathbf{Q}$ is a $6 \times 6$ matrix describing the error covariance matrix of the state vector $\mathbf{x}_{\varepsilon, t}$. Since it is assumed 
that the offset error is not correlated with gravity, the matrix format is described by

$$
\mathbf{Q}_{t}=E\left(\mathbf{x}_{\varepsilon} \mathbf{x}_{\varepsilon}^{T}\right)=\left[\begin{array}{cc}
\mathbf{Q}_{g, t} & \mathbf{0} \\
\mathbf{0} & \mathbf{Q}_{b, t}
\end{array}\right] .
$$

The Kalman filter uses the error model, together with the covariance matrices $\mathbf{Q}$ and $\mathbf{R}$ to attribute the prediction error $\left(\mathbf{y}_{\varepsilon, t}\right)$ of the accelerometer signal to the different accelerometer signal components. $\mathbf{Q}_{t}$ and $\mathbf{R}_{t}$ depend on the covariance matrices of the previously estimated states and the unknown model input signals $\mathbf{w}_{a, t}, \mathbf{w}_{b, t}$ and $\omega_{I I, t}$.

The $3 \times 6$ matrix $\mathbf{C}$ was found by subtracting the predicted sensor output from the actual sensor output

$$
\begin{aligned}
\mathbf{y}_{\varepsilon, t} & =\hat{\mathbf{y}}_{t}^{-}-\mathbf{y}_{t} \\
& =\left(\hat{\mathbf{a}}_{t}^{-}-\hat{\mathbf{g}}_{t}^{-}+\hat{\mathbf{b}}_{t}^{-}\right)-\left(\mathbf{a}_{t}-\mathbf{g}_{t}+\mathbf{b}_{t}+\mathbf{v}_{A, t}\right) \\
& =\left(-\mathbf{g}_{\varepsilon, t}^{-}+\mathbf{b}_{\varepsilon, t}^{-}\right)+\mathbf{a}_{\varepsilon, t}^{-}-\mathbf{v}_{A, t} \\
& =\left[\begin{array}{ll}
-\mathbf{I}_{3} & \mathbf{I}_{3}
\end{array}\right] \cdot \mathbf{x}_{\varepsilon}+\mathbf{v}_{t} .
\end{aligned}
$$

$\mathbf{I}_{3}$ is the $3 \times 3$ identity matrix and the first matrix on the last line of (13) equals $\mathbf{C}$. The noise term $\mathbf{v}_{t}$ incorporates the measurement noise $\mathbf{v}_{A, t}$ and the $a$ priori acceleration error estimate $\mathbf{a}_{\varepsilon}^{-}$.

The matrix A describes the dynamics of the error state. The state only contains prediction errors that do not depend on previous estimates. Therefore, $\mathbf{A}$ equals the zero matrix.

\section{E. Error Behavior}

Finally, an expression of the prediction error covariance matrices $\mathbf{Q}_{t}$ and $\mathbf{R}_{t}$ will be derived. Prediction errors are either caused by errors in previously estimated states or unknown inputs $\mathbf{w}_{a}, \mathbf{w}_{\mathrm{B}}$, and $\boldsymbol{\omega}_{I I}$. A relation is derived that describes the error in the predicted states as a function of the previous estimation errors and unknown inputs. The covariance matrix is then obtained by taking the variance.

The error of the predicted acceleration $\left(\mathbf{a}_{\varepsilon, t}^{-}\right)$expressed in the sensor coordinate frame was found by comparing the estimate (3) with the modeled acceleration (2)

$$
\begin{aligned}
S, t \hat{\mathbf{a}}_{t}^{-} & ={ }^{S, t} \mathbf{a}_{t}+{ }^{S, t} \mathbf{a}_{\varepsilon, t}^{-} \\
& ={ }^{S, t} \mathbf{a}_{t}+\mathrm{H}(\mathrm{q})\left\{{ }^{S, t} \mathbf{a}_{\varepsilon, t-1}\right\}+{ }^{S, t} \mathbf{w}_{a, t} .
\end{aligned}
$$

Using (5) to express the acceleration in the sensor frame and neglecting products of errors, yields the following acceleration error:

$$
\begin{aligned}
{ }^{S, t} \hat{\mathbf{a}}_{t}^{-}= & { }^{S, t-1} \hat{\mathbf{a}}_{t}^{-}-T \hat{\boldsymbol{\omega}}_{t} \times{ }^{S, t-1} \hat{\mathbf{a}}_{t}^{-} \\
= & { }^{S, t} \mathbf{a}_{t}+\left(I-T\left[\hat{\boldsymbol{\omega}}_{t} \times\right]\right)^{S, t-1} \mathbf{a}_{\varepsilon, t-1}^{-} \\
& +\left[{ }^{P} \hat{\mathbf{a}}_{t}^{-} \times\right] T \boldsymbol{\omega}_{\varepsilon, t} .
\end{aligned}
$$

The matrix cross product is defined as

$$
[\boldsymbol{\omega} \times] \cdot \mathbf{x}=\left[\begin{array}{ccc}
0 & -\omega_{z} & \omega_{y} \\
\boldsymbol{\omega}_{z} & 0 & -\boldsymbol{\omega}_{x} \\
-\boldsymbol{\omega}_{y} & \boldsymbol{\omega}_{x} & 0
\end{array}\right] \cdot \mathbf{x}=\boldsymbol{\omega} \times \mathbf{x} .
$$

The estimate of the acceleration is critical in movements with a large centripetal acceleration. Even if the mean of the acceleration in a global coordinate frame is zero it does not mean that the mean acceleration in the sensor coordinate frame is close to zero. Especially in movements with large centripetal accelerations like bending, the acceleration in the sensor frame will not be close to zero. Therefore, the predicted acceleration is rotated every timestep. If either the acceleration or the angular velocity estimation is inaccurate, a biased prediction of acceleration will occur. The effect of an error in acceleration is shown by the second term of (15) and the effect of an angular velocity error is represented by the third term. When the acceleration estimate is biased, it will cause biased offset and gravity estimates.

Neglecting the change by renormalization (4), the prediction error of gravity is determined by the error in the previous gravity estimate and the error introduced by rotating with an angular velocity error. In the same way as was done for the acceleration, the prediction error of the gravity was derived to be

$$
\begin{aligned}
{ }^{S, t} \hat{\mathbf{g}}_{t}^{-}={ }^{S, t} \mathbf{g}_{t}+\left(I-T\left[\hat{\boldsymbol{\omega}}_{t} \times\right]\right)^{S, t-1} \mathbf{g}_{\varepsilon, t-1}^{-} \\
+\left[{ }^{S, t-1} \hat{\mathbf{g}}_{t}^{-} \times\right] T \boldsymbol{\omega}_{\varepsilon, t} .
\end{aligned}
$$

Since the angular velocity can only be estimated in the direction perpendicular to the accelerometer signal and the gravity vector is a large component of this signal, the error in gravity prediction will be relatively small with respect to the acceleration prediction. Since the accuracy of the angular velocity estimate is determined by the change in acceleration, the gravity estimate will be poorer in periods with a relatively large acceleration.

\section{F. Covariances}

The angular velocity error covariance matrix is obtained by taking the variances of the unknown quantities in (8)

$$
\mathbf{Q}_{\omega, t}=Q_{\Omega} \cdot \mathbf{n}_{t} \cdot \mathbf{n}_{t}^{T}+\frac{1}{g_{c}^{2}}\left[\hat{\mathbf{g}}_{t} \times\right] \cdot \mathbf{Q}_{\Delta a} \cdot\left[\hat{\mathrm{g}}_{t} \times\right]^{T} .
$$

$Q_{\Omega}$ is the variance of the actual angular velocity averaged over the $\mathrm{x}, \mathrm{y}$, and $\mathrm{z}$ axes during a measurement, and $\mathbf{Q}_{\Delta a}$ is the covariance matrix describing the change in acceleration. Both are parameters of the Kalman filter which need to be determined to estimate the components of the accelerometer signal.

Taking the variances of (15) yields the acceleration error covariance matrix

$$
\begin{aligned}
\mathbf{Q}_{a, t}=\left(I-T\left[\hat{\boldsymbol{\omega}}_{t} \times\right]\right) & \mathbf{Q}_{a, t-1}\left(I-T\left[\hat{\boldsymbol{\omega}}_{t} \times\right]\right)^{T} \\
& +T^{2}\left[{ }^{S, t} \hat{\mathbf{a}}_{t}^{-} \times\right] \mathbf{Q}_{\omega, t}\left[{ }^{S, t} \hat{\mathbf{a}}_{t}^{-} \times\right]^{T} .
\end{aligned}
$$

In much the same way as was done with the acceleration prediction, the gravity error covariance matrix was found to be

$$
\begin{aligned}
\mathbf{Q}_{g, t}=\left(I-T\left[\hat{\boldsymbol{\omega}}_{t} \times\right]\right) \mathbf{Q}_{g, t-1}\left(I-T\left[\hat{\boldsymbol{\omega}}_{t} \times\right]\right)^{T} & \\
& +T^{2}\left[{ }^{P} \mathbf{g}_{t}^{-} \times\right] \mathbf{Q}_{\omega, t}\left[{ }^{P} \mathbf{g}_{t}^{-} \times\right]^{T} .
\end{aligned}
$$

The covariance of the estimation $\hat{\mathbf{b}}_{t}^{-}$that is made by substituting $\hat{\mathbf{b}}_{t-1}$ with covariance matrix $\mathbf{Q}_{b, t-1}$ into (9) and setting $\mathbf{w}_{b}$ to zero is:

$$
\mathbf{Q}_{b, t}=\mathbf{Q}_{b, t-1}+\mathbf{Q}_{w b} .
$$

$\mathbf{Q}_{w b}$ is the covariance matrix of $\mathbf{w}_{b}$. If the duration of the measurement is only a few minutes or the temperature is relatively 


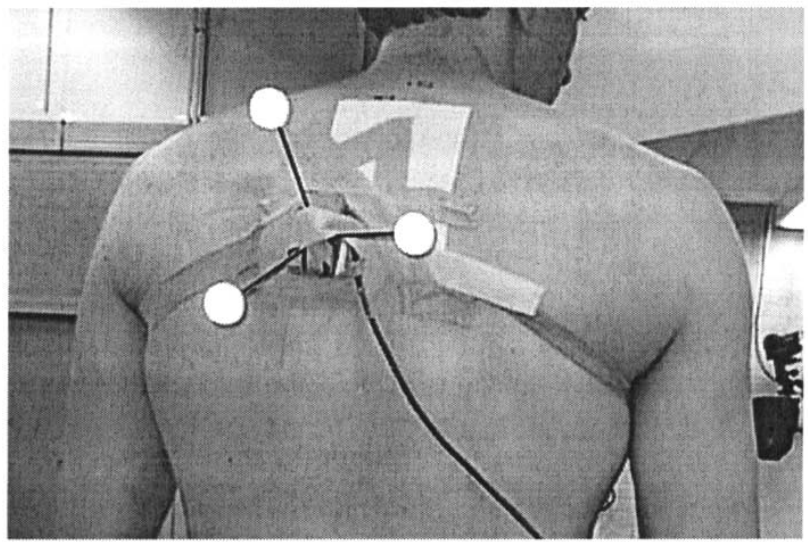

Fig. 3. Placement of the IMU on the back on the trunk. The sensor module is placed at the T4/T5 level just left of the spine in order to mount the sensor on a flat surface. Three reflective markers were placed on the IMU housing in order to measure the reference orientation of the accelerometer using the Vicon system as a reference.

constant, the change in offset will play a minor role with respect to the initial uncertainty.

\section{METHODS: EXPERIMENTAL VALIDATION}

A 3-D accelerometer was assembled using three AD XL-05 single-axis capacitive accelerometers that were mounted perpendicular with respect to each other. In order to compare the estimated angular velocity with the real angular velocity, three orthogonally mounted gyroscopes (Murata, model ENC05) were added as a reference. The resulting inertial measurement unit (IMU) was sized $55 \times 25 \times 30 \mathrm{~mm}$ and weighted $40 \mathrm{~g}$. It was calibrated according to Ferraris [18], enabling the measurement of accelerometer signals and angular velocity in the IMU coordinate frame. The output signals were sampled at $100 \mathrm{~Hz}$ using a portable datalogger (weight $900 \mathrm{~g}$, size $6 \times 10 \times 19 \mathrm{~cm}$ ). The IMU was attached to the body segment using double-sided adhesive tape and Leukoplast (Fig. 3) and mounted with the $z$ axis in cranial direction, the $x$ axis in frontal direction, and the $y$ axis laterally to the left.

A crate-lifting task was chosen to test the algorithm as described in the previous sections. In this task, the subject was asked to move a pile of five empty beer crates one by one from one stack to another, placed $1 \mathrm{~m}$ away at the pace of a metronome. As soon as the stack was empty the subject started stacking the crates vice versa. This task was chosen because of the 3-D nature of the movement and the relevance of the use of accelerometers for back load estimation.

The following two series of crate lifting measurements were conducted:

1) a set of trials for identification of the model parameters, constituting of a series of ten trials of 2 min each at a comfortable lifting speed of 3 s per crate;

2) a series to test the filter performance, constituting of a series of ten trials of 2 min each at different lifting speeds of subsequently $7,6,5,4,3.5,3.5,3,3,2$, and 1.5 crates per second.

Both series were conducted with a different male subject. Both signed an informed consent prior to the measurement. One IMU was placed at the pelvis and one at the trunk at the height of T4/T5.

As a reference, the inclination of the IMU was recorded with a five-camera Vicon optical motion capturing system with a sampling rate of $50 \mathrm{~Hz}$. The system measured the position of three markers that were placed on 100-mm carbon fiber sticks secured to the IMU (Fig. 3). These marker positions were used to construct a marker coordinate frame. An estimate of the accuracy of the marker frame orientation was obtained by analyzing the relative motion of two markers. The root-mean square (rms) of the orientation error was assumed to be in the same order as the rms distance variation divided by the distance between the two markers. The orientation of the IMU coordinate frame with respect to the Vicon markers was found by holding the IMU still for $4 \mathrm{~s}$ in two different orientations.

To identify the parameters for offset fluctuation, it was assumed that it is mainly determined by temperature changes. The effect of temperature on accelerometer offset was measured by cooling two IMUs in an oven from $40{ }^{\circ} \mathrm{C}$ to $20^{\circ} \mathrm{C}$ in a time period of $3 \mathrm{~h}$. This was done with the IMU laying on six different sides, enabling measurement of all accelerometer offsets. A value for a typical accelerometer offset error was obtained using the change in output from $30{ }^{\circ} \mathrm{C}$ to $20^{\circ} \mathrm{C}$, since this was assumed to be a typical temperature step for mounting a calibrated IMU on a body segment.

Model parameters are the coefficients of the polynomial $H(q)$ and the variances of $\mathbf{w}_{a}, \mathbf{w}_{b}$ and $\boldsymbol{\omega}$. The offset was assumed not to change during the 2-min trials and was set to zero. The angular velocity variance was obtained using the gyroscopes. The acceleration parameters in $H(q)$ and $\mathbf{Q}_{a}$ were determined by analyzing the acceleration of the trunk during the stacking task. The acceleration was obtained by adding the gravity to the accelerometer output vector (1). The gravity was obtained using the Vicon reference measurement. It was assumed that the offset $\mathbf{b}_{t}$ and measurement noise vector $\mathbf{v}_{a, t}$ could be neglected for the purpose of identification of the accelerometer spectrum. A fifth-order polynomial was used to describe $H(q)$. Its coefficients were found using the Matlab system identification toolbox developed by Ljung [19].

The inclination was defined as the angle that the negative gravity vector makes with the $z$ axis of the IMU coordinate frame. This definition coincides with the intuitive measure of a water dial. The rms error of the inclination estimate during a trial was taken as a measure of inclination error. In order to compare the effect of the filter with respect to other methods for inclination measurement using an accelerometer, the inclination was obtained from the accelerometer signals using three methods. In the first method, the measured signals were processed using the Kalman filter as described earlier. In the second method, the Kalman filter was used without taking account of changing IMU coordinate frame orientation (5). In this case, the angular velocity estimate was set to zero and its variance was set to $Q_{\Omega}$. The last method did not make use of the Kalman filter, but was based on low-pass filtering the sensor signals using a zero-phase Butterworth filter and considering the direction of the result. The cutoff-frequency was set to $4 \mathrm{~Hz}$ and was determined by plotting a spectrum of the acceleration. 
The accelerometer signals were each low-pass filtered with a cutoff frequency of $4 \mathrm{~Hz}$, using a fourth-order Butterworth filter. The inclination was estimated from the direction of the resulting signal vector.

\section{RESULTS}

The accuracy of the reference measurements using the optokinetic system depends on the accuracy of the position measurement of the markers and on the accuracy of the marker-to-IMU orientation estimate. The accuracy of the position measurement was estimated by considering the distance between two markers. The standard deviation of the fluctuation of measured distance was $1 \mathrm{~mm}$. This corresponds to a standard deviation of measured orientation of less than $1^{\circ}$. The orientation of the marker coordinate frame with respect to the IMU coordinate frame was obtained using the accelerometer signal during two moments in which the IMU was put in different orientations, assuming the accelerometer only measures gravity when holding the accelerometer still and simultaneously measuring the orientation of the marker frame. An error in IMU-to-marker orientation is caused by the variation of the accelerometer offset. The offset change of six accelerometers after a temperature step from $30^{\circ}$ to $20^{\circ}$ was $0.2 \mathrm{~m} / \mathrm{s}^{2}$ on average (s.d. $0.2 \mathrm{~m} / \mathrm{s}^{2}$ ). An offset error of $0.2 \mathrm{~m} / \mathrm{s}^{2}$ corresponds to an angle error of $1.1^{\circ}$. It was assumed that these were the largest sources of error of the orientation obtained using the reference system.

An example of the accelerometer output signals during two crate lifts is given in Fig. 4. At the start of the trial, the subject was in an upright position. At this time, the accelerometer output was approximately $1 \mathrm{~g}$ in the $z$ direction of the IMU coordinate frame, since the $z$ direction of the IMU was chosen to point cranially. The accelerometer output along the IMU $x$ and $y$ axes was close to zero at this time, since these were almost horizontal. Once the subject starts to bend, the IMU $x$ axis starts to point downwards and the IMU $z$ axis turns horizontally. Hence, the change in $x$ and $z$ accelerometer output at 1.5 and $4 \mathrm{~s}$.

The variance value of the white noise term $\mathbf{v}_{a, t}$, estimated by measuring accelerometer output while the IMU is laying still, was $0.1 \mathrm{~m} / \mathrm{s}^{2}$. The error of the angular velocity depends on two effects: the uncertainty of the angular velocity in the direction parallel to the IMU output vector and the errors caused by a change in acceleration. The error due to change in acceleration was given by the second term on the right hand side of (8) and was diminished by low-pass filtering the accelerometer signal before the angular velocity is estimated. To determine the cutoff frequency, the spectrum of the angular velocity magnitude, obtained using gyroscopes, is plotted together with the spectrum of $w_{\varepsilon \perp}$, given in (8) (Fig. 5). Based upon this figure, the cutoff-frequency for determining the angular velocity signal was chosen to be $4 \mathrm{~Hz}$, and a fourth-order zero-phase Butterworth filter was applied.

An example of the estimated angular velocity of the trunk is plotted in Fig. 6. The left figure shows the $y$ component of the angular velocity for bending forward and getting upright, measured using the gyroscopes and the accelerometer. For this movement, the error will be largely determined by a change in acceleration. Mainly because of the centripetal acceleration,

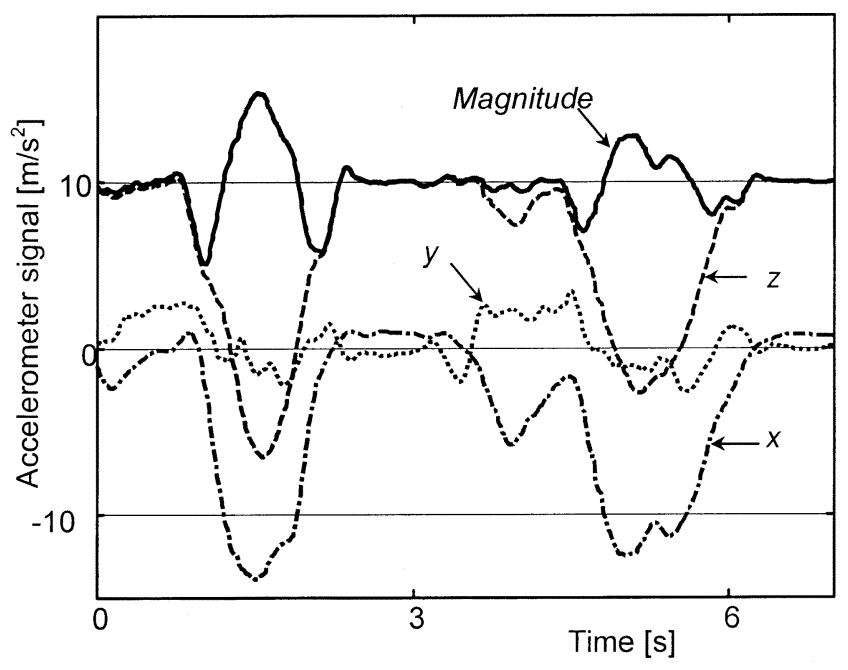

Fig. 4. Example recording of the three accelerometer signals of the trunk accelerometer during two crate lifts. The magnitude of the accelerometer signal vector is given by the solid line.

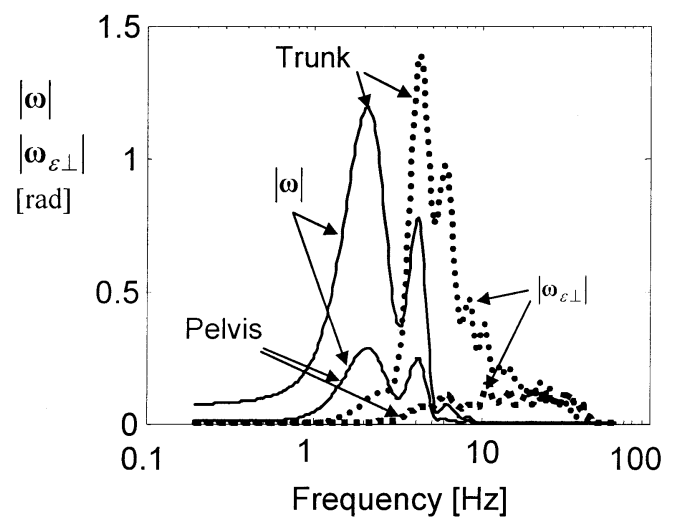

Fig. 5. Spectra of angular velocity $\omega$ (solid) and angular velocity error $\omega_{\varepsilon \perp}$ (dashed) in the direction perpendicular to the accelerometer output vector. The spectra were taken using the gyroscope signals measured during a crate lifting trial at comfortable lifting speed ( $3.5 \mathrm{~s}$ per crate) at the trunk and the pelvis.

the acceleration will be large when the angular velocity is also large. The plot on the right hand side of Fig. 6 shows the angular velocity along the global $Z$ direction as estimated with an accelerometer and as measured using gyroscopes during a torsion trial of the trunk while standing upright. As expected, the angular velocity in the direction of the accelerometer vector $\mathbf{y}_{t}$ can not be measured using an accelerometer.

To assess the possibility of measuring the angular velocity during lifting trials, the rms of the angular velocity error magnitude as a percentage of angular velocity magnitude is given in Fig. 7. The percentages are obtained by averaging the rms of the angular velocity errors over 10 trials at comfortable lifting speed (3 s per crate). As expected, the errors in estimating angular velocity from acceleration are mainly due to the angular velocity component parallel to gravity, which can not be estimated using accelerometers.

The measured and identified spectrum of the acceleration is given in Fig. 8. The acceleration in all three directions is used in the identification process. Due to a limited order of the model, 

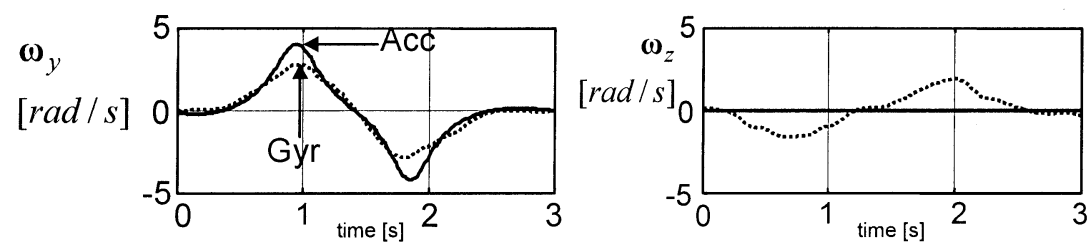

Fig. 6. Angular velocity as measured with accelerometer (solid) and gyroscope (dotted) on the trunk. The left graph shows the angular velocity along the lateral ( $y$ ) axis during a flexion with the accelerometer on the trunk. The right graph shows the angular velocity estimates along the global vertical during a torsion trial.
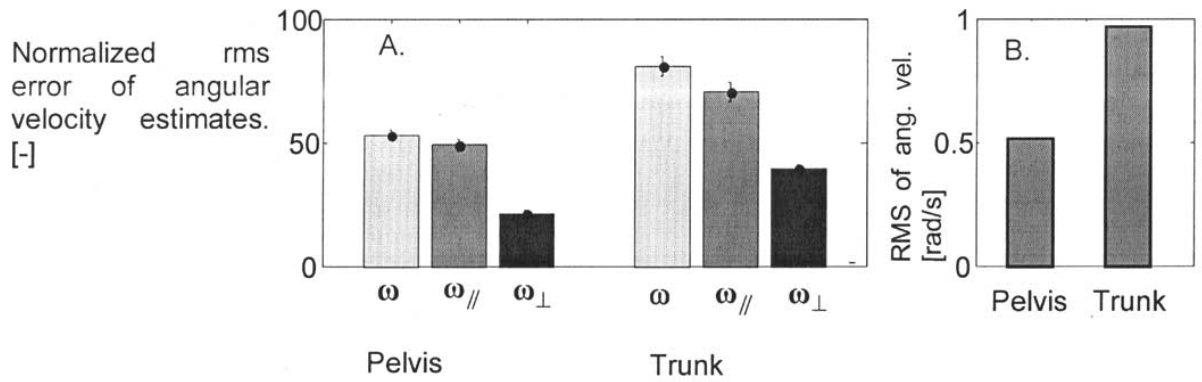

Fig. 7. (A) RMS error of the angular velocity estimates of an accelerometer averaged over ten lifting trials. Errors are given as a percentage of angular velocity magnitude rms. $\boldsymbol{\omega}_{\|}$is the angular velocity error along the accelerometer output vector that cannot be measured using the accelerometer signals. $\boldsymbol{\omega}_{\perp}$ is the angular velocity error in the direction perpendicular to the accelerometer output vector. (B) RMS of the angular velocity magnitude for pelvis and trunk averaged over ten trials.

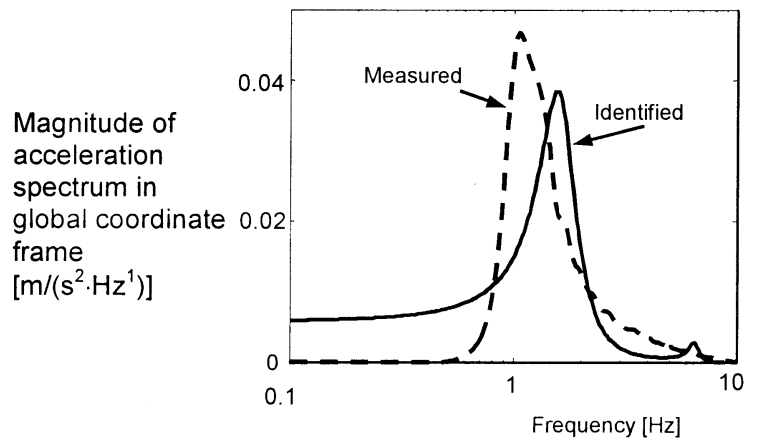

Fig. 8. Measured and identified spectrum of the acceleration in the global frame. The acceleration in all three directions of nine comfortable speed trials was used to identify a fifth-order autoregressive process. The spectrum of this identified system was compared to the spectum that was obtained with a Fourier transform of the same acceleration signals.

the identified spectrum does not exactly coincide with the spectrum that is obtained using a Fourier transformation. Since a least squares identification of a time series will generally focus on prediction of the high-frequent components, mainly the spectrum estimate of the low-frequent components differs considerably with the spectrum obtained by Fourier transformation.

\section{A. Inclination Estimate by the Kalman Filter}

The ability of the filter to estimate inclination with and without taking account of changing orientation of the IMU coordinate frame as assessed by the estimated angular velocity was compared to the performance of the inclination estimate based on a low-pass filtering of all three accelerometer signals (Fig. 9). The rms of the inclination error using the filter was significantly smaller as compared to the inclination error obtained by the method of only low-pass filtering the accelerometer signals. Accounting for rotation did not significantly improve the inclination estimate.

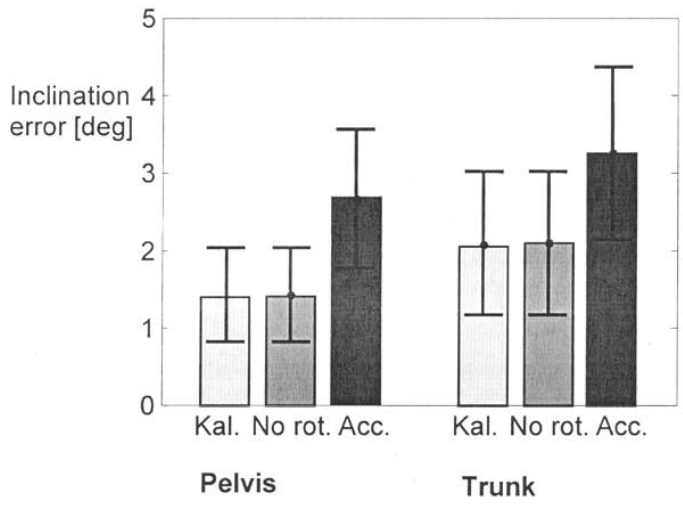

Fig. 9. Average rms inclination error. Kal: estimated using the Kalman filter. No rot: estimated using the Kalman filter, not accounting for changing sensor frame orientation, by setting the angular velocity to zero. Acc: estimated using the accelerometer as an inclinometer, applying a cutoff freuqency of $4 \mathrm{~Hz}$ to all three accelerometer signals. The inclination errors were averaged over ten trials for the Pelvis and nine trials for the trunk sensor.

To test the effect of speed of movement on the inclination estimation, the rms of the inclination error of each trial was plotted against lifting speed (Fig. 10). A linear regression was used to describe the effect of lifting speed on inclination estimation errors. All graphs had a slope significantly different from zero $(p<0.05)$. Again, the Kalman filter performed better in estimating the pelvis as well as trunk inclination and this difference tended to increase at higher lifting speeds.

\section{B. Offset}

An example of the accelerometer offset estimation is given in Fig. 11. It shows the magnitude of the offset error vector when the accelerometer signals were processed with the Kalman filter using an initial offset error of $1 \mathrm{~m} / \mathrm{s}^{2}$ in $x$ direction. Each of the two lines represents a trial at a different lifting speed. After a rapid decline of offset error during the first few seconds, the 

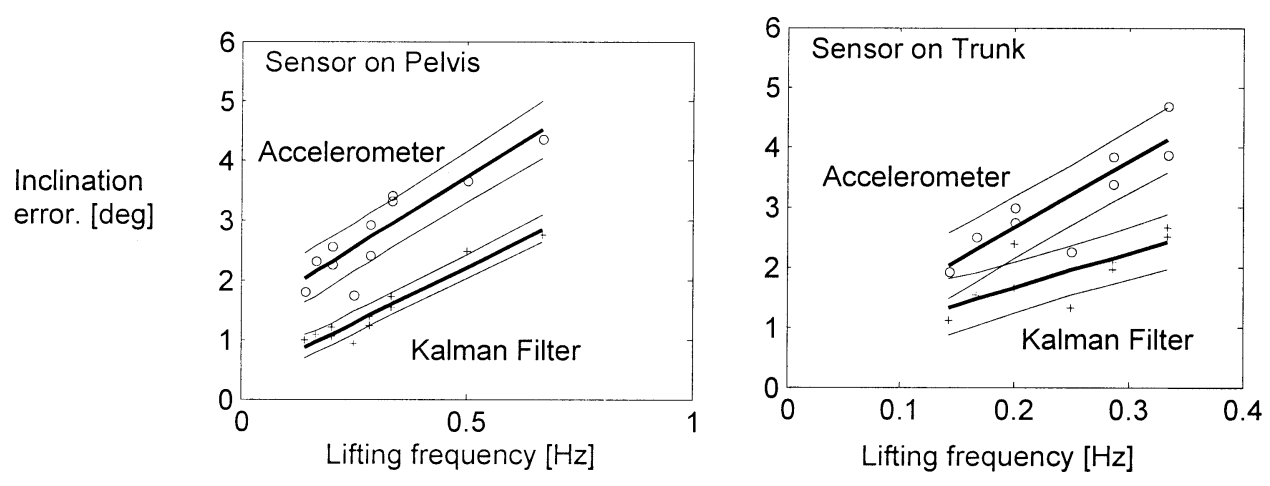

Fig. 10. Error in estimated inclination against the number of crates lifted per second, obtained using a Kalman filter and using the accelerometer directly. The accelerometer was placed on the (left) pelvis and (right) trunk.

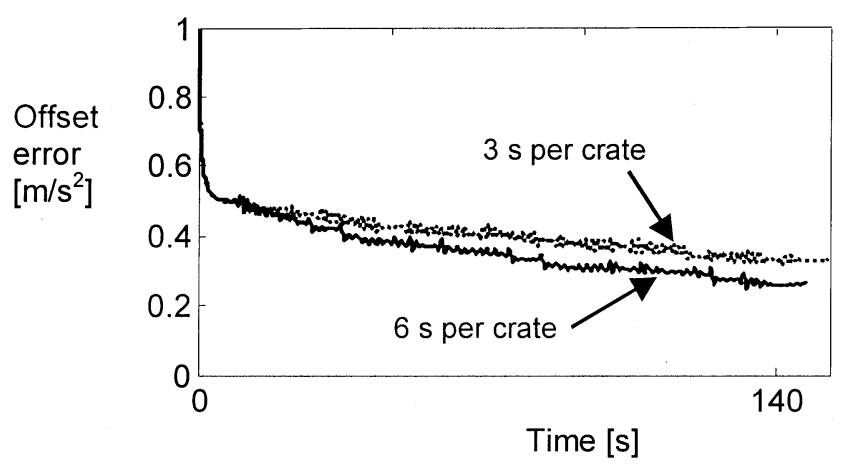

Fig. 11. Estimation of the magnitude of the accelerometer offset error vector during lifting trials at the speed of $3 \mathrm{~s}$ per crate and the speed of $6 \mathrm{~s}$ per crate. Offset is given an initial error of $1 \mathrm{~m} / \mathrm{s}^{2}$ in the $x$ direcion (forward).

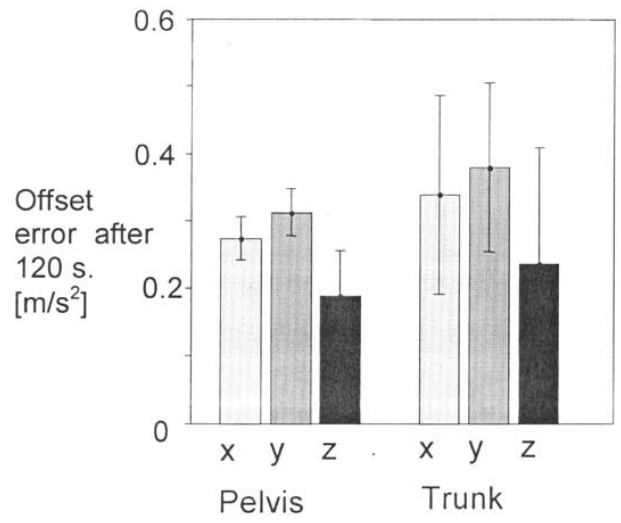

Fig. 12. Average magnitude of offset error vector after $120 \mathrm{~s}$ of crate lifting at different lifting speeds, subsequently applying an initial offset error of $1 \mathrm{~m} / \mathrm{s}^{2}$ in each of the three sensor axes $(x, y$, and $z)$. Values were averaged over ten trials.

offset error monotonically decreases. There is only a small difference between offset estimation during a slow and a fast task.

The effect of an initial offset on different axes was investigated by artificially adding an offset error of $1 \mathrm{~m} / \mathrm{s}^{2}$ to one of the IMU axes. The offset estimate at the end of the 120-s trial was taken as a measure of the ability of the filter to estimate the offset. The results are shown in Fig. 12. Using a paired $t$-test, it was found that the different estimates were not significantly different from each other. It appeared that an offset in the IMU $z$ axis could be estimated with a smaller error than the $y$ and $x$ axes. This is because the offset can only be estimated in the direction of gravity, and the sensor $z$ axis was pointing in the gravity direction more often than the $x$ and $y$ axes of the IMU.

\section{DISCUSSION}

\section{A. Inclination}

Two important assumptions in the design of the Kalman filter are: the acceleration has a bandpass characteristic and the acceleration is zero in the global coordinate frame. The effect of these assumptions was tested by comparing the filter performance to 1) the filter performance used without taking account of changing IMU coordinate frame orientation (Fig. 9) and 2) to the performance of direct inclination estimation from the accelerometer signal vector after lowpass filtering all three constituting signals. Of these three methods, the two approaches using a Kalman filter perform almost twice as good as the method based on a lowpass filtered accelerometer signal. The two different versions of the Kalman filter did not differ significantly. Taking account of changing sensor coordinate frame orientation between subsequent measurement instances using the estimated angular velocity does not substantially improve the performance and it can, therefore, be concluded that the improvement of the Kalman filter over the lowpass filter can be solely attributed to the assumption that the acceleration is described by a bandpass characteristic.

The conclusion that taking into account the angular velocity does not improve the filter can be explained by the poor estimation of the angular velocity (Fig. 7). An advantage of not using the angular velocity information in the Kalman filter is that the algorithm can be implemented in real time. Since the zero phase filter requires samples in advance, the angular velocity can not be obtained in real time. Without using the a priori estimated angular velocity, the Kalman filter can be implemented in real time.

The way the sensor is fixed to the subject may be of influence on the accelerations the sensor experiences. When the sensor unit is mounted to a rigid object like an orthosis, high-frequent accelerations can be measured, especially during shocks. These accelerations are unlikely to cause large errors in measured inclination, since the filter will attribute high-frequent sensor signals to either acceleration or noise instead of an inclination change. 


\section{B. Offset}

The mean acceleration of the sensor in an earth-fixed coordinate frame is assumed to be zero in all directions. Because of centripetal accelerations, this is generally not the case for the acceleration with respect to the sensor frame. If the angular velocity is not estimated correctly, the predicted acceleration and, hence, the offset will be biased. Another factor that may determine the offset accuracy is the angle over which the sensor is rotated. Since the offset can only be estimated in gravity direction, the offset estimate in all three directions will be more accurate when the sensor is rotated over a larger angle.

From Fig. 12, it can be seen that an offset error in cranial direction can be reduced more rapidly than in a horizontal direction, reflecting that the offset can only be estimated in gravity direction. The reason that the pelvis offset estimation was somewhat better than that of the trunk may be attributed to the centripetal accelerations that are larger on the trunk. Apparently, the larger orientation changes of the trunk do not compensate for this.

\section{Task}

The sensor was tested on the pelvis and trunk because these positions are relevant for the measurement of back load and stability [8]. The task that was tested will probably give larger errors than a task involving a sequence of daily tasks because it involves a continuous repeated movement. The filter is expected to be more accurate in periods involving small accelerations and angular velocities, like many daily life activities (Fig. 10). These periods hardly occur in the considered crate lifting task, especially at high frequencies of performing the task. If the subject is in a moving vehicle, the sensor can experience low-frequent accelerations. The effect of such low-frequent accelerations will be a temporal error in the measured inclination. The estimation of the accelerometer offset will be hardly influenced provided that the offset change is much slower than the period of the accelerations of the vehicle.

The offset estimation is expected to be relatively accurate during periods with relatively small acceleration and angular velocity, especially in the vertical direction. These periods occur frequently during daily life. It is to be expected that the Kalman filter will accurately estimate the offset for long recordings of the daily life of patients. In these recordings, automatic recalibration is relevant because frequent calibration of the accelerometer is difficult. The effect of an offset error of $1 \mathrm{~m} / \mathrm{s}^{2}$ in a horizontal direction will cause an inclination error of nearly $6^{\circ}$, and will, therefore, be a significant error in many applications. The described Kalman filter may significantly improve robustness of such systems. Especially when an accelerometer is to be implanted, robustness is an important specification.

If the Kalman filter is applied to accelerometer measurements on other segments like arms or legs, moving with large centripetal acceleration components, the inclination estimate will probably be less accurate.

\section{CONCLUSION}

The proposed Kalman filter is able to estimate inclination of trunk and pelvis with an error of $2^{\circ}$ RMS for the functional 3-D movements which have been evaluated. This is nearly twice as accurate as an estimate obtained by low-pass filtering of the accelerometer signals. A more accurate estimate of the inclination using ambulatory methods can be obtained using additional sensors like gyroscopes [20]-[22]. However, current micromachined gyroscopes are heavier, larger, require more power than state of the art micromachined accelerometers. For this reason, only accelerometers may be used if weight and power are important. Even when small, relatively inaccurate accelerometers are used, the described method yields a robust method for long term recording of human movement.

\section{REFERENCES}

[1] P. H. Veltink, H. B. Bussmann, W. de Vries, W. L. Martens, and R. C. van Lummel, "Detection of static and dynamic activities using uniaxial accelerometers," IEEE Trans. Rehab. Eng., vol. 4, pp. 375-385, Dec. 1996.

[2] H. J. Busser, W. G. de Korte, E. B. Glerum, and R. C. van Lummel, "Method for objective assessment of physical work load at the workplace," Ergonomics, vol. 41, pp. 1519-1526, 1998.

[3] F. Foerster, M. Smeja, and J. U. Fahrenberg, "Detection of posture and motion by accelerometry: A validation study in ambulatory monitoring," Comput. Hum. Behav., vol. 15, pp. 571-583, 1999.

[4] C. V. C. Bouten, K. T. M. Koekkoek, M. Verduin, R. Kodde, and J. D. Janssen, "A triaxial accelerometer and portable processing unit for the assessment of daily physical activity," IEEE Trans. Biomed. Eng., vol. 44, pp. 136-147, Mar. 1997.

[5] G. Uswatte, "Objective measurement of functional upper-extremity movement using accelerometer recordings transformed with a treshold filter," Stroke, vol. 31, pp. 662-667, 2000.

[6] R. Moe-Nilssen, "A new method for evaluating motor control in gait under real-life environmental conditions. Part 1: The instrument," Clin. Biomech., vol. 13, pp. 320-327, 1998.

[7] A. J. van den Bogert, L. Read, and B. M. Nigg, "A method for inverse dynamic analysis using accelerometry," J. Biomech., vol. 29, pp. 949-954, 1996.

[8] C. T. M. Baten, P. Oosterhoff, I. Kingma, P. H. Veltink, and H. J. Hermens, "Inertial sensing in ambulatory load estimation," in Proc. IEEE Engineering in Medicine and Biology Soc. 18th Annu. Int. Conf., Amsterdam, The Netherlands, 1996.

[9] N. Fisekovic and D. B. Popovic, "New controller for functional electrical stimulation systems," Med. Eng. Phys., vol. 23, pp. 391-399, 2001.

[10] A. T. Willemsen, F. Bloemhof, and H. B. Boom, "Automatic stanceswing phase detection from accelerometer data for peroneal nerve stimulation," IEEE Trans. Biomed. Eng., vol. 37, pp. 1201-1208, Dec. 1990.

[11] K. Y. Tong and M. H. Granat, "Virtual artificial sensor technique for functional electrical stimulation," Med. Eng. Phys., vol. 20, pp. 458-468, 1998.

[12] J. Lötters, W. Olthuis, P. H. Veltink, and P. Bergveld, "Design, realization and characterization of a symmetrical triaxial capacitive accelerometer for medical applications," Sens. Actuat. A, vol. 61, pp. 303-308, 1997.

[13] B. Kemp, A. J. M. W. Janssen, and B. van der Kamp, "Body position can be monitored in 3-D using miniature accelerometers and earth-magnetic field sensors," Electroencephalogr. Clin. Neurophysiol./Electromyogr. Motor Contr., vol. 109, pp. 484-488, 1998.

[14] J. Lötters, J. Schipper, P. Veltink, W. Olthuis, and P. Bergveld, "In-use calibration procedure for a triaxial accelerometer," Sens. Actuat. A, vol. 68, pp. 221-228, 1998.

[15] G. Kamen, C. Patten, C. D. Du, and S. Sison, "An accelerometry-based system for the assessment of balance and postural sway," Gerontology, vol. 44, pp. 40-45, 1998.

[16] R. G. Brown and P. Y. C. Hwang, Introduction to Random Signals and Applied Kalman Filtering. $\quad$ New York: Wiley, 1997.

[17] R. E. Kalman, "A new approach to linear filtering and prediction problems,” J. Basic Eng., pp. 35-45, 1960.

[18] F. Ferraris, U. Grimaldi, and M. Parvis, "Procedure for effortless in-field calibration of three-axis rate gyros and accelerometers," Sens. Mater., vol. 7, pp. 311-330, 1995. 
[19] L. Ljung, System Identification, Theory for the User. Englewood Cliffs, NJ: Prentice-Hall, 1999.

[20] R. E. Mayagoitia, A. V. Nene, and P. H. Veltink, "Accelerometer and rate gyroscope measurement of kinematics: An inexpensive alternative to optical motion analysis systems," J. Biomech., vol. 35, pp. 537-542, 2002.

[21] E. R. Bachman, "Inertial and magnetic tracking of limb segment orientation for inserting humans in synthetic environments," $\mathrm{Ph}$.D. disseration, Naval Postgrad. School, Monterey, CA, 2000.

[22] H. J. Luinge, Inertial Sensing of Human Movement. Enschede, The Netherlands: Twente Univ. Press, 2002.

Henk J. Luinge received the M.Sc. and Ph.D. degrees in mechanical engineering from the University of Twente, Enschede, The Netherlands, in 1998 and 2002, respectively. His Ph.D. research was on the topic of inertial sensing of human movement.

After working at Xsens Technologies B.V., Enschede, The Netherlands, he began a postdoctoral fellowship in the Sensory Motor Performance Program, Rehabilitation Institute of Chicago, Chicago, IL.
Peter H. Veltink (S'85-M'88) received the M.Sc. and Ph.D. degrees in electrical engineering from the University of Twente, Enschede, The Netherlands, in 1984 and 1988, respectively. His Ph.D. degree research was in the area of electrical nerve stimulation.

Currently, he is a Professor of technology for the restoration of human function at the Institute for Biomedical Technology, University of Twente, and performs research in the area of artificial motor control and ambulatory sensory systems with applications to rehabilitation medicine. He is the Scientific Coordinator of the European Union training networks NEUROS (1996-2000) and NeuralPRO (2000-2004). He has been the Project Leader for the University of Twente in several European projects, including the BIOMED2 Sensation project, CREST project, and Concerted Actions RAFT. He performed sabbaticals at Case Western Reserve University, Cleveland, OH, in 1989 and at the Center for Sensory-Motor-Interaction, Aalborg University, Aalborg, Denmark, in 1997.

Dr. Veltink received the Royal Shell Stimulating Prize for his contribution to the rehabilitation engineering field in 1997. He was the Treasurer of the International Functional Electrical Stimulation Society from 1996 to 2001. 